Pacific

Journal of

Mathematics

NONEXISTENCE RESULTS AND CONVEX HULL PROPERTY FOR MAXIMAL SURFACES IN MINKOWSKI THREE-SPACE

Rosa MARIA BARReiro Chaves AND LeONOR FerRer 


\title{
NONEXISTENCE RESULTS AND CONVEX HULL PROPERTY FOR MAXIMAL SURFACES IN MINKOWSKI THREE-SPACE
}

\author{
Rosa MARIA BARREIRO CHAVES AND LEONOR FERRER
}

\begin{abstract}
We study properly immersed maximal surfaces with nonempty boundary and singularities in three-dimensional Minkowski space. We use the maximum principle and scaling arguments to obtain nonexistence results for these surfaces when the boundary is planar. We also give sufficient conditions for such surfaces to satisfy the convex hull property.
\end{abstract}

\section{Introduction}

In recent years, maximal hypersurfaces in a Lorentzian manifold - that is, spacelike submanifolds of codimension one with zero mean curvature - have been the object of considerable interest. Such hypersurfaces, and in general those having constant mean curvature, have a special significance in classical relativity [Marsden and Tipler 1980].

When the ambient space is the flat Minkowski space $\mathbb{L}^{n+1}$, Calabi [1970] (for $n \leq 3$ ) and Cheng and Yau [1976] (for arbitrary dimension) proved that a complete maximal hypersurface is necessarily a spacelike hyperplane. This result remains valid if we replace the completeness hypothesis by properness; see [Fernández and López 2004b]. Therefore, it does not make sense to consider global problems on regular maximal hypersurfaces in $\mathbb{L}^{n+1}$. Interesting problems are then those that deal with hypersurfaces with nonempty boundary or having certain type of singularities. In this line, Bartnik and Simon [1982/83] obtained results on the existence and regularity of spacelike solutions to the boundary value problem for the mean curvature operator in $\mathbb{L}^{n+1}$, and Kobayashi [1984] investigated surfaces with conelike singularities. Estudillo and Romero [1992] defined a class of maximal surfaces with singularities of other types and studied criteria for such a surface to be a plane. On the other hand, Klyachin and Mikyukov [1993] have tackled the problem of existence of solutions to the maximal hypersurface equation in $\mathbb{L}^{n+1}$ with prescribed boundary conditions and a finite number of singularities. Fernández, López and Souam [Fernández et al. 2005] proved that a complete embedded maximal surface

MSC2000: primary 53C50; secondary 53C42, 53C 80.

Keywords: maximal surfaces.

Research partially supported by MCYT-FEDER grant number MTM2004-00160. 
with a finite set of singularities is an entire graph over any spacelike plane and that this family of maximal graphs has a structure of moduli space. We also mention the work of Umehara and Yamada [2006] where topological obstructions to the existence of this type of surfaces are given.

Maximal surfaces in $\mathbb{L}^{3}$ and minimal surfaces in Euclidean space are closely related. Both are solutions of variational problems, namely they are local maxima (minima) for the area functional. Both admit a Weierstrass representation (see [Kobayashi 1983] for maximal surfaces). The maximal surface equation and the minimal surface equation are both quasilinear elliptic equations and therefore enjoy a maximum principle. But contrary to the minimal case, solutions to the maximal surface equation can have isolated singularities, that is to say, points where the solution is not differentiable. Such points correspond to possible degeneracy of the ellipticity of the maximal surface equation. Geometrically at these singular points the Gauss curvature blows up, the Gauss map has no well-defined limit and the surface is asymptotic to the light cone.

In the minimal case, the maximum principle has been used by Schoen [1983], Hoffman and Meeks [1990], Meeks and Rosenberg [1993], López and Martín [2001], and others to derive remarkable results. In this paper we apply the maximum principle and scaling arguments to properly immersed maximal surfaces with nonempty boundary and isolated singularities in $\mathbb{L}^{3}$. We get two types of results: nonexistence results for properly immersed maximal surfaces with singularities and planar boundary contained in a timelike or lightlike plane, and results generalizing the convex hull property for such surfaces. Recall that a surface satisfies the convex hull property if it lies in the convex hull of its boundary. Although compact maximal surfaces in $\mathbb{L}^{3}$ satisfy this property, since they have nonpositive euclidean Gauss curvature (see [Osserman 1971/72]), this is not true if compactness is not assumed. We give sufficient conditions for a properly immersed maximal surface (not necessarily compact and with singularities) to satisfy the convex hull property.

Organization of paper. Section 2 contains the necessary notations and definitions, a description of the behavior of maximal surfaces around an isolated singularity, and a discussion of the maximal surfaces we use as barriers: Lorentzian catenoids, maximal surfaces of Riemann and Scherk type, and spacelike planes. We finish the section giving a first generalization of the convex hull property to compact maximal surfaces with singularities.

In Section 3 we obtain nonexistence results for properly immersed maximal surfaces with singularities and boundary contained in a timelike plane. Letting

$$
\mathrm{C}^{+}=\left\{\left(x_{1}, x_{2}, x_{3}\right) \in \mathbb{R}^{3} \mid x_{1}^{2}+x_{2}^{2}-x_{3}^{2} \leq 0, x_{3} \geq 0\right\}
$$

be the positive solid half-cone, we show: 
Theorem A. There exists no connected properly immersed maximal surface $M$ such that $M \subset\left\{\left(x_{1}, x_{2}, x_{3}\right) \in \mathbb{R}^{3} \mid x_{2} \geq 0,-a x_{2}+x_{3} \geq 0\right\}$ and $\partial M \subset C^{+} \cap$ $\left\{\left(x_{1}, x_{2}, x_{3}\right) \in \mathbb{R}^{3} \mid x_{2}=0\right\}$, for $\left.a \in\right]-1,1[$.

This theorem holds even if we allow certain singularities (see Theorem 3.5).

Section 4 is devoted to the study of properly immersed maximal surfaces whose boundary is contained in a spacelike plane. Consider any region $V$ of the form

$$
V=\left\{\left(x_{1}, x_{2}, x_{3}\right) \in \mathbb{R}^{3} \mid x_{3} \geq 0,-a x_{2}+x_{3} \leq 0, x_{1}+b x_{2}+c \geq 0\right\},
$$

with $a \in] 0,1[$ and $b, c \in]-\infty, \infty[$.

Theorem B. Let $M$ be a connected properly immersed maximal surface contained in $V$ and such that $\partial M$ lies in a spacelike plane. Then $M$ is a planar region.

This result, too, holds even if we allow certain singularities (see Theorem 4.2 and Corollary 4.3). In the proof we construct a barrier surface ad hoc using the aforementioned Bartnik and Simon existence result. Theorem B is still valid if we replace $V$ by $\mathrm{C}^{+}$(see Proposition 4.4).

Finally, in Section 5 we exploit the results of the preceding sections to give nonexistence results for properly immersed maximal surfaces with the boundary on a lightlike plane. We also prove:

Theorem C. Any connected properly immersed maximal surface with singularities contained either in $\mathrm{V}$ or $\mathrm{C}^{+}$lies in the convex hull of its boundary and some of its singularities.

Propositions 5.3 and 5.4 provide a precise formulation of this result.

\section{Preliminaries}

We denote by $\llbracket^{3}$ the three dimensional Lorentz-Minkowski space $\left(\mathbb{R}^{3},\langle\rangle,\right)$, where the inner product corresponds to the form $d x_{1}^{2}+d x_{2}^{2}-d x_{3}^{2}$. A nonzero vector $v \in \mathbb{R}^{3}$ is called spacelike, timelike or lightlike if $\langle v, v\rangle$ is positive, negative or zero, respectively. The vector $(0,0,0)$ is considered spacelike. We say that a plane in $\mathbb{L}^{3}$ is spacelike, timelike or lightlike if the induced metric is Riemannian, nondegenerate and indefinite or degenerate, respectively. We also say that an affine plane in $\mathbb{L}^{3}$ is spacelike, timelike or lightlike if it is parallel to a spacelike, timelike or lightlike vector plane.

The light cone at $y=\left(y_{1}, y_{2}, y_{3}\right) \in \mathbb{L}^{3}$ is defined as

$$
C(y)=\left\{x \in \mathbb{L}^{3} \mid\langle x-y, x-y\rangle=0\right\} .
$$

We also set $C^{+}(y)=C(y) \cap\left\{x_{3} \geq y_{3}\right\}$ and $C^{-}(y)=C(y) \cap\left\{x_{3} \leq y_{3}\right\}$. Observe that lightlike vectors in $\mathbb{L}^{3}$ lie in the light cone $C((0,0,0))$. 
Further, set $\mathbb{W}^{2}=\mathbb{U}_{+}^{2} \cup \mathbb{T}_{-}^{2}$, where $\mathbb{U}_{+}^{2}=\left\{x \in \mathbb{L}^{3} \mid\langle x, x\rangle=-1\right\} \cap\left\{x_{3} \geq 0\right\}$ and $\mathbb{Q}_{-}^{2}=\left\{x \in \mathbb{L}^{3} \mid\langle x, x\rangle=-1\right\} \cap\left\{x_{3} \leq 0\right\}$.

Consider the stereographic projection $\sigma: \overline{\mathbb{C}}-\{|z|=1\} \rightarrow \mathbb{M}^{2}$ for $\mathbb{H}^{2}$, given by

$$
\sigma(z)=\left(\frac{2 \operatorname{Im} z}{|z|^{2}-1}, \frac{2 \operatorname{Re} z}{|z|^{2}-1}, \frac{|z|^{2}+1}{|z|^{2}-1}\right),
$$

where $\overline{\mathbb{C}}=\mathbb{C} \cup\{\infty\}$ and $\sigma(\infty)=(0,0,1)$.

An immersion $X: M \rightarrow \mathbb{L}^{3}$ is spacelike if the tangent plane at any point is spacelike. In this case $M$ must be orientable, that is to say, the Gauss map $N$ is globally well defined and $N(M)$ lies in one of the components of $\mathbb{T}^{2}$.

A maximal immersion is a spacelike immersion $X: M \rightarrow \mathbb{L}^{3}$ whose mean cur-

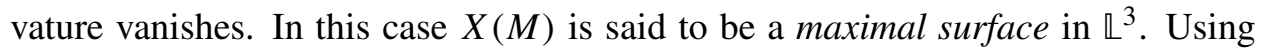
isothermal parameters compatible with a fixed orientation $N: M \rightarrow \mathbb{H}^{2}, M$ acquires a natural conformal structure, and the map $g=\sigma^{-1} \circ N$ is meromorphic. Moreover, there exists a holomorphic 1-form $\Phi_{3}$ on $M$ such that the 1-forms

$$
\Phi_{1}=\frac{i}{2}\left(\frac{1}{g}-g\right) \Phi_{3}, \Phi_{2}=-\frac{1}{2}\left(\frac{1}{g}+g\right) \Phi_{3}
$$

are holomorphic, and together with $\Phi_{3}$, have no real periods on $M$ and no common zeros. Up to a translation, the immersion is given by

$$
X=\operatorname{Re} \int\left(\Phi_{1}, \Phi_{2}, \Phi_{3}\right) .
$$

The induced Riemannian metric $d s^{2}$ on $M$ is given by $d s^{2}=\lambda\left(d u^{2}+d v^{2}\right)$, where $z=u+i v$ is a conformal parameter and

$$
\lambda=\frac{1}{2}\left(\left|\Phi_{1}\right|^{2}+\left|\Phi_{2}\right|^{2}-\left|\Phi_{3}\right|^{2}\right)=\left(\frac{\left|\Phi_{3}\right|}{2}\left(\frac{1}{|g|}-|g|\right)\right)^{2} .
$$

Since $M$ is spacelike, we have $|g| \neq 1$ on $M$ and we can assume $|g|<1$.

Conversely, let $M, g$ and $\Phi_{3}$ be a Riemann surface, a meromorphic map on $M$ and a holomorphic 1-form on $M$. If $|g(p)| \neq 1$ for all $p \in M$, and if the 1-forms $\Phi_{1}$, $\Phi_{2}, \Phi_{3}$ defined as above are holomorphic, have no real periods and no common zeros, then the conformal immersion $X$ defined in (2-3) is maximal and its Gauss map is $\sigma \circ g$. We call $\left(M, g, \Phi_{3}\right)$ the Weierstrass representation of $X$. For more details see [Kobayashi 1983].

A maximal surface in $\mathbb{L}^{3}$ can be represented locally as a graph $x_{3}=u\left(x_{1}, x_{2}\right)$ of a smooth function $u$ such that $u_{x_{1}}^{2}+u_{x_{2}}^{2}<1$ and

$$
\left(1-u_{x_{1}}^{2}\right) u_{x_{2} x_{2}}+2 u_{x_{1}} u_{x_{2}} u_{x_{1} x_{2}}+\left(1-u_{x_{2}}^{2}\right) u_{x_{1} x_{1}}=0 .
$$

The maximum principle for elliptic quasilinear equations then gives rise to: 
Maximum principle for maximal surfaces. Let $S_{1}$ and $S_{2}$ be two maximal surfaces in $\mathbb{L}^{3}$ which intersect tangentially at a point $p \in S_{1} \cap S_{2}$. Suppose that $u_{i}$, for $i=1,2$ denotes the function defining $S_{i}$ around $p$ and that $u_{1} \geq u_{2}$ (we say $S_{1}$ is above $S_{2}$ or $S_{2}$ is below $S_{1}$ ). Then $S_{1}=S_{2}$ locally around $p$.

Maximal surfaces with singularities. If in a maximal immersion $X: M \rightarrow \mathbb{1}^{3}$ we allow points $q \in M$ where the induced metric is not Riemannian we say that $X$ (respectively, $X(M)$ ) has singularities and $q$ (respectively, $X(q)$ ) is called a singular point. The different kinds of isolated singularities of maximal surfaces and the behavior of maximal surfaces around these points are well known; see [Kobayashi 1984; Ecker 1986; Miklyukov 1992; Fernández et al. 2005]. We recall the necessary material.

Let $D$ be an open disc and $X: D \rightarrow \mathbb{R}^{3}$ a maximal immersion with a singular point in $q \in D$. There are two possibilities: either $N$ extends continuously to $q$ ( $q$ is a spacelike singular point) or not ( $q$ is a lightlike singular point).

In the second case $D-\{q\}$ with the induced metric is conformally equivalent to $\{z \in \mathbb{C}, 0<r<|z|<1\}$ and $X$ extends to a conformal map $X: A_{r} \rightarrow \mathbb{L}^{3}$ with $X\left(\mathbb{S}^{1}\right)=X(q)=p$, where $A_{r}=\{z \in \mathbb{C}, r<|z| \leq 1\}$ and $\mathbb{S}^{1}=\{z \in \mathbb{C},|z|=1\}$. Denote by $J(z)=1 / \bar{z}$ the inversion about $\mathbb{S}^{1}$. Then Schwarz reflection allows us to assert that $X$ extends analytically to $B_{r}=\{z \in \mathbb{C}, r<|z|<1 / r\}$ and satisfies $X \circ J=-X+2 p$. Therefore if $\left(g, \Phi_{3}\right)$ are the Weierstrass data of the extended immersion we have $J^{*}\left(\Phi_{k}\right)=-\bar{\Phi}_{k}$ for $k=1,2,3$, where $J^{*}\left(\Phi_{k}\right)$ denotes the pullback of $\Phi_{k}$ under $J$ : if $\Phi_{k}=f_{k} d z$ then $J^{*}\left(\Phi_{k}\right)=-\bar{z}^{-2}\left(f_{k} \circ J\right) d \bar{z}$. Thus $g \circ J=1 / \bar{g}$ and consequently $|g|=1$ on $\mathbb{S}^{1}$. Let $\Pi$ be a spacelike plane containing

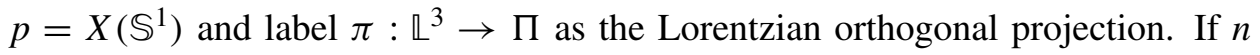
(always even) is the number of zeros of $\Phi_{3}$ on $\mathbb{S}^{1}$ and $m$ denotes the degree of the map $g: \mathbb{S}^{1} \rightarrow \mathbb{S}^{1}$, we have:

Lemma 2.1 [Fernández et al. 2005]. There exists a small closed disc $U$ in $\Pi$ centered at $p$ such that $(\pi \circ X)^{-1}(p) \cap V=\mathbb{S}^{1}$ and $(\pi \circ X): V-\mathbb{S}^{1} \rightarrow U-\{p\}$ is a covering of $m+\frac{1}{2} n$ sheets, where $V$ is the annular connected component of $(\pi \circ X)^{-1}(U)$ containing $\mathbb{S}^{1}$.

As a consequence, $X$ is an embedding around $q$ if and only if $m=1$ and $n=0$. In this case the point $p=X\left(\mathbb{S}^{1}\right)$ is said to be a conelike singularity of the maximal surface $X(D)$. Moreover, for $r_{0}$ close enough to $1, X\left(A_{r_{0}}\right)$ is the graph of a function $u$ over $\Pi$. Locally, conelike singularities are points where the function defining the graph is not differentiable and correspond to possible degeneracy of the equation (2-4). Moreover, the graph of $u$ is either above $\Pi$ and asymptotic to $C^{+}(p)$ or below $\Pi$ and asymptotic to $C^{-}(p)$, and the point $p$ is called a downward or upward pointing conelike singularity, respectively. 


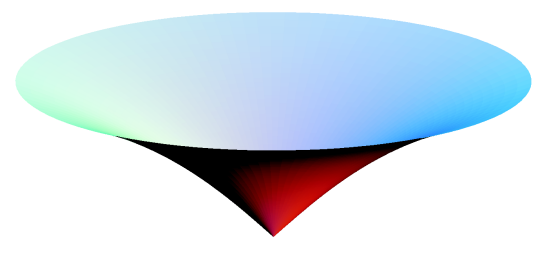

(a)

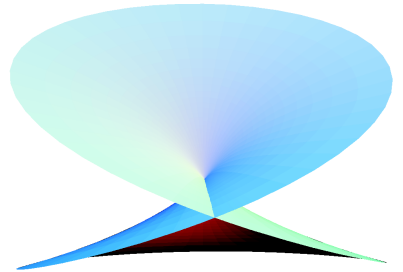

(b)
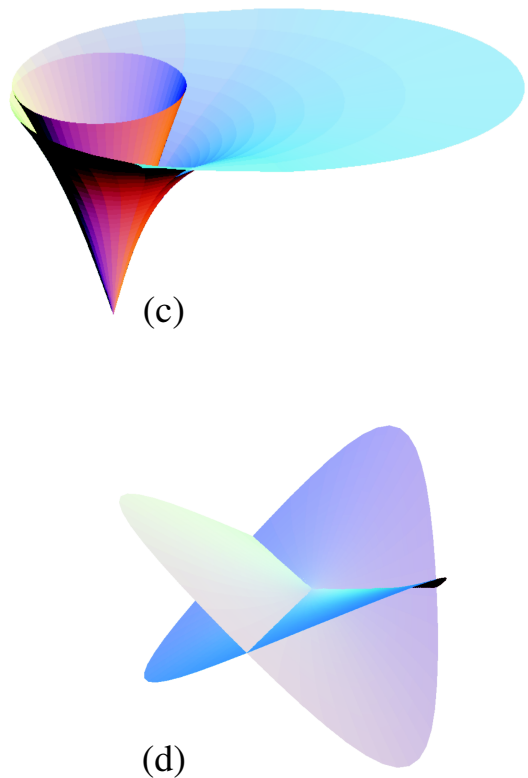

(d)

Figure 1. Different types of isolated singularities. (a) A downward pointing conelike singularity $(m=1, n=0)$. (b) a downward pointing lightlike singularity with $m=2, n=0$. (c) a lightlike singularity with $m=1, n=2$. (d) a spacelike singularity with $n=2$.

Lemma 2.2. Let $D$ be an open disc and $X: D \rightarrow \mathbb{L}^{3}$ a maximal immersion with a lightlike singular point in $q \in D$. Set $p=X(q)$. The neighborhoods $U$ and $V$ of Lemma 2.1 can be chosen so that:

(i) If $p$ is a lightlike singularity with $n=0$, then $X(V)$ is either over $\Pi$ and asymptotic to $C^{+}(p)$ or below $\Pi$ and asymptotic to $C^{-}(p)$ (see Figure 1a,b).

(ii) If, on the contrary, $p$ is a lightlike singularity with $n>0$, there exist points of $X(V)$ in both sides of the plane $\Pi$. In particular there exist a pair of curves $\alpha$, $\beta$ in $V$ starting at $q$ such that $X(\alpha)-\{p\}$ is over $\Pi$ and asymptotic to $C^{+}(p)$ and $X(\beta)-\{p\}$ is below $\Pi$ and asymptotic to $C^{-}(p)$ (see Figure $1 \mathrm{c}$ ).

Proof. Up to a Lorentzian isometry we can assume $\Pi=\left\{x_{3}=0\right\}$ and $p=(0,0,0)$. Let $X: A_{r} \rightarrow \mathbb{R}^{3}$ be a conformal reparametrization of the maximal immersion with $X\left(\mathbb{S}^{1}\right)=p$ and consider $U, V$ as in Lemma 2.1. A thoughtful reading of the proof of Lemma 2.1 in [Fernández et al. 2005] will convince the reader that the same arguments prove (i). 
For the proof of (ii) we use again the ideas of the same lemma. The Weierstrass data can be written in a neighborhood of $\mathbb{S}^{1}$ as

$$
g(z)=z^{m}, \quad \Phi_{3}(z)=i \frac{\prod_{j=1}^{n}\left(z-a_{j}\right)}{z^{n / 2+1}} f(z) d z,
$$

where $a_{1}, \ldots, a_{n}$ are the zeros of $\Phi_{3}$ on $\mathbb{S}^{1}$ (with multiplicity) and $f$ is a nonvanishing holomorphic function. Recall that the multiplicity of the zero of $\Phi_{3}$ at $a_{i}$ coincides with the number of nodal curves of the harmonic function $x_{3}$ meeting at $a_{i}$ minus one. By the maximum principle there are no domains bounded by nodal curves and $x_{3}$ changes sign when crossing a nodal curve. Since $n \geq 2$ there are points of $X(V)$ in both sides of $\Pi$ and there exist at least a pair of domains $\Gamma, \Gamma^{\prime} \subset V$ bounded by a pair of nodal curves of $x_{3}$, a piece of $\partial V-\mathbb{S}^{1}$ and a point or a piece of $\mathbb{S}^{1}$, such that $x_{3}(X(\Gamma))>0$ and $x_{3}\left(X\left(\Gamma^{\prime}\right)\right)<0$.

To conclude we prove that the image of all the curves $\rho_{\theta}(s)=s e^{i \theta}$, for $\theta \in$ $K=[0,2 \pi]-\left\{\arg \left(a_{1}\right), \ldots, \arg \left(a_{n}\right)\right\}$, is asymptotic to the cone $C(p)$. Taking into account (2-5) we can write

$$
\begin{aligned}
& X\left(\rho_{\theta}(s)\right)=\operatorname{Re} \int_{1}^{s} \frac{i \prod_{j=1}^{n}\left(t e^{i \theta}-a_{j}\right)}{t^{n / 2+1}\left(e^{i \theta}\right)^{n / 2}} f\left(t e^{i \theta}\right) \\
& \quad \times\left(\frac{i}{2}\left(\frac{e^{-i m \theta}}{t^{m}}-t^{m} e^{i m \theta}\right),-\frac{1}{2}\left(\frac{e^{-i m \theta}}{t^{m}}+t^{m} e^{i m \theta}\right), 1\right) d t .
\end{aligned}
$$

Since $J^{*}\left(\Phi_{3}\right)=-\bar{\Phi}_{3}$, we deduce that

$$
\operatorname{Im}\left(\frac{i \prod_{j=1}^{n}\left(e^{i \theta}-a_{j}\right)}{\left(e^{i \theta}\right)^{\frac{n}{2}}} f\left(e^{i \theta}\right)\right)=0 .
$$

Using this it is straightforward to see that

$$
\lim _{s \rightarrow 1}\left\|\frac{X\left(\rho_{\theta}(s)\right)}{x_{3}\left(X\left(\rho_{\theta}(s)\right)\right)}-(\sin (m \theta),-\cos (m \theta), 1)\right\|_{1}=0,
$$

where $\|\cdot\|_{1}$ is the $\mathscr{C}^{1}$ norm in $\mathscr{C}^{1}\left(K, \mathbb{R}^{3}\right)$. Therefore, we can consider a pair of curves $\alpha \in \Gamma$ and $\beta \in \Gamma^{\prime}$ satisfying the requirements of statement (ii).

Definition 2.3. A point $p$ as in Lemma 2.2(i) is called a downward or upward pointing lightlike singularity, as the case may be. We also call it a general conelike singularity.

If $D$ is an open disc and $X: D \rightarrow \mathbb{L}^{3}$ is a maximal immersion with a spacelike singular point in $q \in D$, the local behavior at the singularity is similar to the case of minimal surfaces in $\mathbb{R}^{3}$ (see [Dierkes et al. 1992; Estudillo and Romero 1992; Fernández et al. 2005]): $X$ is not a topological embedding, $D-\{q\}$ with the induced metric is conformally equivalent to $\{z \in \mathbb{C}|0<| z \mid<1\}$, the Weierstrass data $\left(g, \Phi_{3}\right)$ 
extend analytically to $q,|g(q)|<1$ and $\Phi=\left(\Phi_{1}, \Phi_{2}, \Phi_{3}\right)$ has a zero at $q$. Up to a Lorentzian isometry we can assume that the tangent plane of $X(D)$ at $p=X(q)$ is $\Pi=\left\{x_{3}=0\right\}$ and $p=(0,0,0)$. The Weierstrass data of the immersion can be written as

$$
g(z)=z^{m} f(z), \quad \Phi_{3}(z)=z^{m+n} d z
$$

where $m>0, n$ is the order of the zero of $\Phi$ at $q$ and $f$ is a holomorphic function with $f(0) \neq 0$. Up to a rotation around the axis $x_{3}$, we can assume $\operatorname{Im}(f(0))=0$. From here it is easy to derive that the asymptotic behavior of the immersion around the singularity is in polar coordinates

$$
\begin{aligned}
X\left(s e^{i \theta}\right)= & \left(\frac{-s^{n+1}}{2 f(0)(n+1)} \sin ((n+1) \theta)+O\left(s^{n+2}\right),\right. \\
& \left.\frac{-s^{n+1}}{2 f(0)(n+1)} \cos ((n+1) \theta)+O\left(s^{n+2}\right), \frac{s^{m+n+1}}{m+n+1} \cos ((m+n+1) \theta)\right),
\end{aligned}
$$

where by $O\left(s^{n+2}\right)$ we denote a function such that $s^{-n-2} O\left(s^{n+2}\right)$ is bounded as $s \rightarrow 0$. Therefore, it is clear that $X$ has a branch point at $q$ of order $n$ in the sense of [Gulliver et al. 1973].

Lemma 2.4 [Gulliver et al. 1973, Lemma 2.12]. Let $X: D \rightarrow \mathbb{L}^{3}$ be a maximal immersion with a spacelike singular point at $q \in D$. Set $p=X(q)$ and let be $S$ an embedded surface in $\mathbb{L}^{3}$ with $p \in S$. Suppose that for a neighborhood $V$ of $q$, $X(V)$ lies on one side of $S$. Then the tangent plane to $S$ at $p$ coincides with the tangent plane to $X(D)$ at $p$.

Remark 2.5. In the case of spacelike singularities, we always assume that the immersion $X: D \rightarrow \mathbb{L}^{3}$ is not a branched covering of an embedded surface; that is to say, $q$ is not a false branch point. See [Gulliver et al. 1973].

Finally, we mention a property of maximal surfaces with singularities (see for example [Fernández and López 2004a]).

Lemma 2.6. Let $X: M \rightarrow \mathbb{L}^{3}$ be a maximal immersion with isolated singularities. Then for all $q \in M$ there exists a neighborhood $V$, such that $X(V)-\{X(q)\}$ is contained in the exterior of $C(X(q))$.

Remark 2.7. Let $S$ be an embedded surface and $p \in S$. If the tangent plane of $S$ at $p$ is spacelike then $S$ can be written in a neighborhood of $p$ as the graph of a function $h$ on a domain $\Omega$ of the plane $\left\{x_{3}=0\right\}$. Let $M$ be another surface (possibly with singularities) and denote by $\pi$ the orthogonal projection on $\left\{x_{3}=0\right\}$. In this context, we say that $M$ lies above $S$ in a neighborhood of $p$ if $x_{3}\left(p^{\prime}\right) \geq h\left(x_{1}, x_{2}\right)$ for all $\left(x_{1}, x_{2}\right) \in \Omega$ and $p^{\prime} \in M \cap \pi^{-1}\left(x_{1}, x_{2}\right)$. Naturally, $M$ lies below $S$ if $x_{3}\left(p^{\prime}\right) \leq$ $h\left(x_{1}, x_{2}\right)$ instead. 
Maximal surfaces with boundary. Let $S^{\prime}$ be a maximal surface, possibly with isolated singularities. Consider $S \subset S^{\prime}$ such that the topological boundary of $S$ in $S^{\prime}$ is nonempty and piecewise $\mathscr{C}^{1}$. Then $S$ is called a maximal surface with boundary; the topological boundary of $S$ in $S^{\prime}$ is called the boundary of $S$, written $\partial S$. The interior of $S$ is Int $S=S-\partial S$. Our definition allows singularities on the boundary of $S$.

Since the components of a maximal immersion are harmonic functions, the intersection of such an $S$ with any plane $\Pi$ having $\partial S$ entirely to one side is a union of piecewise analytic curves, and each connected component of $S-(S \cap \Pi)$ is itself a maximal surface with boundary.

We say that $S$ is a properly immersed maximal surface with boundary if, in the preceding situation, $S^{\prime}$ is a maximal surface properly immersed in $\mathbb{L}^{3}$.

Theorem [Fernández and López 2004b]. Let M be a properly immersed maximal surface with boundary such that, except for a compact set, it is contained in the region $\left\{x \in \mathbb{L}^{3} \mid\langle x, x\rangle \geq \varepsilon\right\}$, for $\varepsilon>0$. Then $M$ is relative parabolic, it is to say, bounded harmonic functions on $M$ are determined uniquely by their values at the boundary and the interior isolated singularities.

(Note that in [Fernández and López 2004b] the definition of a maximal surface with boundary is more general than in this paper.)

Corollary 2.8. Let $M$ be a connected properly immersed maximal surface with boundary such that $M \subset\left\{\left(x_{1}, x_{2}, x_{3}\right) \in \mathbb{R}^{3} \mid 0 \leq x_{3} \leq k\right\}$ and the boundary and the singularities are contained in $\left\{\left(x_{1}, x_{2}, x_{3}\right) \in \mathbb{R}^{3} \mid x_{3}=k\right\}$, for $k>0$. Then $M$ is a planar region.

Barrier surfaces. For any $v \in \mathbb{R}^{3}-\{(0,0,0)\}$ and $y \in \mathbb{R}^{3}$, define

$$
\begin{aligned}
H(y, v) & =\left\{x \in \mathbb{R}^{3} \mid\langle v, x-y\rangle_{e}=0\right\}, \\
H^{+}(y, v) & =\left\{x \in \mathbb{R}^{3} \mid\langle v, x-y\rangle_{e} \geq 0\right\}, \\
H^{-}(y, v) & =\left\{x \in \mathbb{R}^{3} \mid\langle v, x-y\rangle_{e} \leq 0\right\},
\end{aligned}
$$

where $\langle,\rangle_{e}$ is the Euclidean metric on $\mathbb{R}^{3}$. Next, for $\theta \in\left[-\frac{\pi}{4}, \frac{\pi}{4}\right]$ and $t \in \mathbb{R}$, set

$$
\begin{aligned}
& \Pi_{\theta t}=H((0,0, t),(0,-\tan \theta, 1)), \\
& \Pi_{\theta t}^{+}=H^{+}((0,0, t),(0,-\tan \theta, 1)), \\
& \Pi_{\theta t}^{-}=H^{-}((0,0, t),(0,-\tan \theta, 1)) .
\end{aligned}
$$

In the case of $t=0$ we write simply $\Pi_{\theta}$ instead of $\Pi_{\theta 0}$, and so on. 
We also consider, for $\alpha \in]-\frac{\pi}{4}, \frac{\pi}{4}[$,

$$
\begin{aligned}
\Sigma_{\alpha} & =H((0,0,0),(0,1,-\tan \alpha)), \\
\Sigma_{\alpha}^{+} & =H^{+}((0,0,0),(0,1,-\tan \alpha)), \\
\Sigma_{\alpha}^{-} & =H^{-}((0,0,0),(0,1,-\tan \alpha)) .
\end{aligned}
$$

Observe that $\Pi_{\pi / 4, t}$ and $\Pi_{-\pi / 4, t}$ are lightlike planes, while the $\Pi_{\theta t}$ are spacelike planes for $\theta \in]-\frac{\pi}{4}, \frac{\pi}{4}[$. Also, for any $\theta \in]-\frac{\pi}{4}, \frac{\pi}{4}[$, there is an orthochronous hyperbolic rotation $f_{s}$ of $\mathbb{L}^{3}$ of the form

$$
f_{s}\left(\begin{array}{l}
x_{1} \\
x_{2} \\
x_{3}
\end{array}\right)=\left(\begin{array}{ccc}
1 & 0 & 0 \\
0 & \cosh s & \sinh s \\
0 & \sinh s & \cosh s
\end{array}\right)\left(\begin{array}{l}
x_{1} \\
x_{2} \\
x_{3}
\end{array}\right),
$$

which preserves (individually) the light half-cones $C^{+}((0,0,0))$ and $C^{-}((0,0,0))$ and satisfies $f_{s}\left(\Pi_{\theta}\right)=\Pi_{0}$. Analogously, for $\left.\theta \in\right]-\frac{\pi}{4}, \frac{\pi}{4}[$, there is an orthochronous isometry $\widetilde{f}_{s}$ of $\mathbb{Q}^{3}$ composed of an orthochronous hyperbolic rotation $f_{s}$ and a vertical translation, and such that $\widetilde{f}_{s}\left(\Pi_{\theta t}^{+}\right)=\Pi_{0}^{+}$and $\widetilde{f}_{s}\left(\Pi_{\theta t}^{-}\right)=\Pi_{0}^{-}$, so also $\widetilde{f}_{s}\left(\Pi_{\theta t}\right)=$ $\Pi_{0}$. As for the $\Sigma_{\alpha}$, they are timelike planes and there is an orthochronous hyperbolic rotation $f_{s}$ of $\mathbb{L}^{3}$ that preserves the light half-cones and satisfies $f_{s}\left(\Sigma_{\alpha}\right)=\Sigma_{0}$. For more details about these isometries of $\mathbb{\complement}^{3}$, see [Fernández and López 2004a].

Now we present the maximal surfaces that we use as barriers.

Lorentzian catenoids. The (vertical) Lorentzian catenoid $\mathscr{C}_{a}$ is the maximal surface given on $\overline{\mathbb{D}}-\{0\}=\{z \in \mathbb{C}|0<| z \mid \leq 1\}$ by the Weierstrass data $g=z$ and $\Phi_{3}=a d z / z$ (see figure for the case $a=1$ ). We can express $\mathscr{C}_{a}$ as the graph of the radially symmetric function

$$
u(r)=-\int_{0}^{r} \frac{a}{\sqrt{t^{2}+a^{2}}} d t, \quad r>0 .
$$

Let $\mathscr{C}=\left\{\mathscr{C}_{a}, a \in\right] 0, \infty[\}$ be the family of such catenoids. Lorentzian catenoids have been used as barriers for applications of the maximum principle in [Bartnik and Simon 1982/83] and [Ecker 1986].

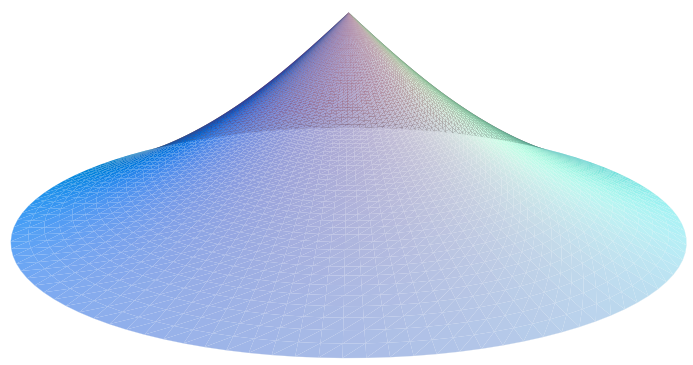


Maximal surfaces of Riemann type. R. López, F. J. López and R. Souam studied in [López et al. 2000] the set of maximal surfaces in $\mathbb{L}^{3}$ that are foliated by pieces of circles. From among them, we take the one-parameter family of Riemanntype maximal surfaces. This is a family of singly periodic maximal surfaces that plays the same role that Riemann's minimal examples play in Euclidean space, and whose fundamental piece is a graph over a spacelike plane, having one planar end and two conelike singularities:

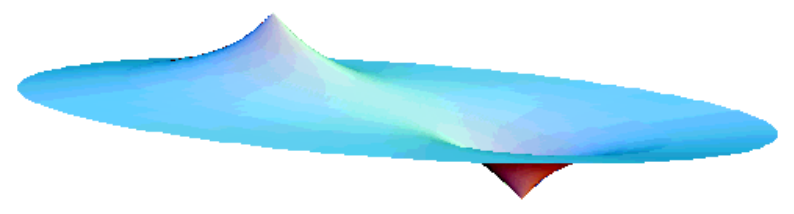

We recall the Weierstrass representation of one-half of a fundamental piece of such surfaces. For $r \in] 1, \infty[$, consider the four-punctured torus

$$
\mathcal{N}=\left\{(z, w) \in \mathbb{C}^{*} \times \mathbb{C} \mid w^{2}=z\left(z^{2}+2 r z+1\right)\right\}
$$

and define in the $z$-plane

$$
s_{0}=\{z \in \mathbb{C}|| z \mid=1\}, \quad s_{1}=\left[r_{1}, 0\left[\times\{0\}, \quad s_{2}=\right]-\infty, r_{2}\right] \times\{0\},
$$

where $r_{1}=-r+\sqrt{r^{2}-1}$ and $r_{2}=-r-\sqrt{r^{2}-1}$. Observe that $r_{2}<-1<r_{1}<0$. Define $N \subset \mathcal{N}$ as the connected component of $z^{-1}\left(\mathbb{C}-\bigcup_{i=0}^{2} s_{i}\right)$ containing the point

$$
\left(\frac{1}{2}, \sqrt{\frac{5}{8}+\frac{1}{2} r}\right)
$$

Finally set $M=\bar{N}$, the closure of $N$ in $\mathcal{N}$.

For brevity, when $z\left(z^{2}+2 r z+1\right) \in \mathbb{R}^{+}$, we set

$$
z_{+}=\left(z,+\sqrt{z\left(z^{2}+2 r z+1\right)}\right), \quad z_{-}=\left(z,-\sqrt{z\left(z^{2}+2 r z+1\right)}\right) .
$$

On $M$ we consider the Weierstrass data $g=z$ and $\Phi_{3}=d z / w$ and the 1-forms $\Phi_{j}, j=1,2$ given by (2-2). The lift $\gamma$ of $s_{0}$ to $M$ generates $\mathscr{H}_{1}(M, \mathbb{Z})$. It is not difficult to see that $\Phi_{1}$ is exact and that $\Phi_{2}, \Phi_{3}$ have no real periods on $\gamma$, so we can consider the maximal immersion $X=\left(X_{1}, X_{2}, X_{3}\right)=\operatorname{Re} \int_{z_{0}}^{z}\left(\Phi_{1}, \Phi_{2}, \Phi_{3}\right)$.

Denote by $\gamma_{1}$ the lift to $M$ of $s_{1}$. It is not hard to prove that $X\left(\gamma_{1}\right)$ is a line parallel to $\left\{x_{2}=x_{3}=0\right\}$. The set of singularities of the immersion is the trace of $\gamma$, and the image of these points under the immersion $X$ is a single point, which we label $P^{r}$. We can choose $z_{0}$ so that $X\left(\gamma_{1}\right)=\left\{x_{2}=x_{3}=0\right\}$ and $P^{r}=\left(0, P_{2}^{r}, P_{3}^{r}\right)$.

Let $\Theta(r) \in\left[-\pi, \pi\left[\right.\right.$ be the angle between $(0,1,0)$ and the vector $P^{r}$, given by

$$
\cos \Theta(r)=\frac{P_{2}^{r}}{\sqrt{\left(P_{2}^{r}\right)^{2}+\left(P_{3}^{r}\right)^{2}}}, \quad \sin \Theta(r)=\frac{P_{3}^{r}}{\sqrt{\left(P_{2}^{r}\right)^{2}+\left(P_{3}^{r}\right)^{2}}} .
$$


To use the surfaces of this family as barriers, we study the function $\Theta(r)$. We have $\Theta(r)=\arctan (h(r) / d(r))$, where

$$
h(r)=X_{3}\left(-1_{-}\right)-X_{3}\left(r_{1}\right)=X_{3}\left(1_{+}\right)-X_{3}(0), \quad d(r)=X_{2}\left(-1_{-}\right)-X_{2}\left(r_{1}\right) .
$$

Hence

$$
\begin{aligned}
& h(r)=\operatorname{Re} \int_{r_{1}}^{-1_{-}} \Phi_{3}=\int_{-1}^{r_{1}} \frac{d t}{\sqrt{t\left(t^{2}+2 r t+1\right)}}, \\
& h(r)=\operatorname{Re} \int_{0}^{1_{+}} \Phi_{3}=\int_{0}^{1} \frac{d t}{\sqrt{t\left(t^{2}+2 r t+1\right)}}, \\
& d(r)=\operatorname{Re} \int_{r_{1}}^{-1_{-}} \Phi_{2}=-\frac{1}{2} \int_{-1}^{r_{1}} \frac{\left(1+t^{2}\right) d t}{t \sqrt{t\left(t^{2}+2 r t+1\right)}} .
\end{aligned}
$$

Since $h$ and $d$ are positive functions, $\Theta(r)$ lies in $] 0, \frac{\pi}{2}[$. Moreover,

$$
d(r)=r h(r)+I(r),
$$

where

$$
I(r)=\frac{1}{2} \int_{-1}^{r_{1}} \frac{\sqrt{t\left(t^{2}+2 r t+1\right)} d t}{t^{2}} .
$$

From (2-7) and (2-8) we see that $\lim _{r \rightarrow 1} \Theta(r)=\frac{\pi}{4}$ and $\lim _{r \rightarrow+\infty} \Theta(r)=0$. From (2-6) we observe that

$$
h^{\prime}(r)=\int_{0}^{1} \frac{-t^{2} d t}{\left(t\left(t^{2}+2 r t+1\right)\right)^{3 / 2}} .
$$

On the other hand, from (2-7) and (2-8) the derivative of $d$ respect to $r$ is

$$
d^{\prime}(r)=r h^{\prime}(r)+\frac{3}{2} h(r) .
$$

According to (2-7) and (2-10) we have

$$
\Theta^{\prime}(r)=\frac{h^{\prime}(r) d(r)-h(r) d^{\prime}(r)}{h(r)^{2}+d(r)^{2}}=\frac{I(r) h^{\prime}(r)-\frac{3}{2} h(r)^{2}}{h(r)^{2}+d(r)^{2}} .
$$

Taking into account (2-8) and (2-9) we get $\Theta^{\prime}(r)<0$, so $\Theta$ is a one-to-one function $\Theta:] 1, \infty[\rightarrow] 0, \frac{\pi}{4}[$.

For $\delta \in] 0, \frac{\pi}{4}$ [ we shall denote by $R_{\delta}$ the maximal surface with boundary defined in $\mathbb{L}^{3}$ by the above immersion for $r=\Theta^{-1}(\delta)$ (see figure at the top of next page). We also set

$$
\mathscr{R}=\left\{R_{\delta} \mid \delta \in\right] 0, \frac{\pi}{4}[\} .
$$

Finally, we need to prove that $R_{\delta} \subset \Pi_{\delta}^{-} \cap\left\{x_{3} \geq 0\right\}$. It is not difficult to see that the point $\{0\}$ is a planar end of the surface asymptotic to the plane $\left\{x_{3}=0\right\}$. Therefore, 


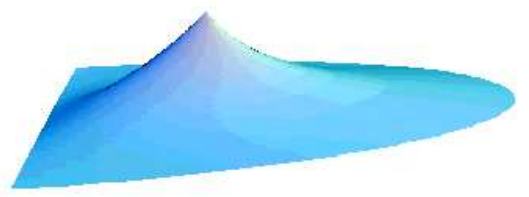

$$
R_{\delta} \text { for } \delta=0.595881 \text {. }
$$

$X_{3}$ is bounded on $M$. From Corollary 2.8 we deduce that $R_{\delta} \subset\left\{x_{3} \geq 0\right\}$. Moreover, from the above facts there exists $t>0$ such that $R_{\delta} \subset \Pi_{\delta t}^{-}$. The maximum principle allows us to assert that $R_{\delta} \subset \Pi_{\delta}^{-}$.

Maximal surfaces of Scherk type. This family of singly periodic maximal surfaces of Scherk type was studied in depth in [Fernández and López 2004a], although an example had already appeared in [Kobayashi 1984]. For $b \in$ ]0, 1[, consider the maximal surface given on $\overline{\mathbb{D}}-\{b,-b\}$ by the Weierstrass data $g(z)=i z$ and

$$
\Phi_{3}(z)=\frac{z d z}{\left(z^{2}-b^{2}\right)\left(b^{2} z^{2}-1\right)} .
$$

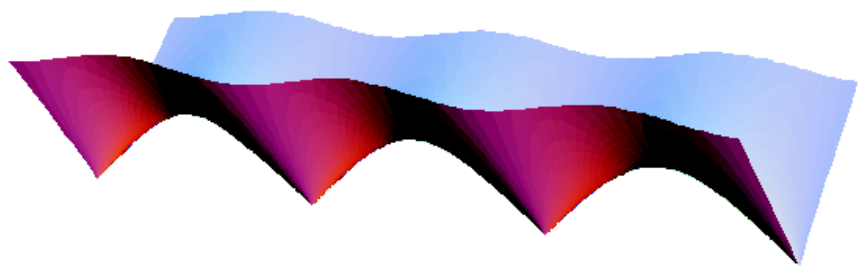

The surface is a graph over a spacelike plane, it is invariant under translation by $\left(0, \pi /\left(2 b\left(b^{2}+1\right)\right), 0\right)$, and each fundamental piece of it has a conelike singularity. Up to translation we can assume that one of these singularities is at $(0,0,0)$, and then all the conelike singularities lie on the line $\left\{x_{1}=x_{3}=0\right\}$. The ends are asymptotic to the totally geodesic horizontal half-cylinder $\partial W_{\delta}$, where

$$
W_{\delta}=\left\{\left(x_{1}, x_{2}, x_{3}\right) \in \mathbb{R}^{3} \mid-\tan \delta x_{1}+x_{3} \geq 0, \tan \delta x_{1}+x_{3} \geq 0\right\},
$$

for $\left.\delta=\arctan \left(2 b /\left(1+b^{2}\right)\right) \in\right] 0, \frac{\pi}{4}[$; for this reason we denote this Scherk-type surface by $S_{\delta}$. By Corollary $2.8, S_{\delta}$ lies entirely in $W_{\delta}$.

The convex hull property. We now prove that a compact maximal surface, even one with isolated singularities, satisfies the convex hull property, that is, it lies in the convex hull of its boundary plus singularities. We will need the following version of the maximum principle for maximal surfaces with singularities. We would like to point out that the proof is inspired in the work [Gulliver et al. 1973].

Proposition 2.9. Let $X: D \rightarrow \mathbb{L}^{3}$ be a maximal immersion with an isolated singular point in $q \in D$. Set $p=X(q)$ and let $S$ be an embedded maximal surface (without singularities) in $\llbracket^{3}$ with $p \in S$. 
(i) If $X(D)$ is above $S$, then $p$ is a downward pointing lightlike singularity.

(ii) If $X(D)$ is below $S$, then $p$ is an upward pointing lightlike singularity.

Proof. We prove (i); the proof of part (ii) is similar. Suppose first that $p$ is a lightlike singularity but not downward pointing. Denote by $\Pi$ the tangent plane to $S$ at $p$ that is a spacelike plane. From Lemma 2.2 we obtain a curve in $X(D)$ asymptotic to $C^{-}(p)$. Since $S$ is asymptotic to $\Pi$ in a neighborhood of $p$ we deduce that there are points of $X(D)$ below $S$ and this contradicts our assumptions.

Now assume $p$ is a spacelike singularity. By Lemma 2.4, the tangent plane to $X(D)$ at $p$ coincides with the tangent plane to $S$ at $p$. Denote this plane by $\Pi$ and by $\pi$ the Lorentzian orthogonal projection on $\Pi$. Up to a Lorentzian isometry we can suppose that $p=(0,0,0)$ and $\Pi=\Pi_{0}$. Consider a disk $\Delta$ in $\Pi$ centered at $(0,0)$ such that $S$ is the graph of a function $h$ on $\Delta$ and $\Delta \subset \pi(X(D))$. Set $M=X(V)$, where $V$ is the connected component of $(\pi \circ X)^{-1}(\Delta)$ containing $q$. If $\partial(M) \cap h(\Delta) \neq \varnothing$, we have an interior regular point in $X(D) \cap S$. By applying the maximum principle we obtain $M=h(\Delta)$ and then $h(\Delta)$ must contain a spacelike singularity. Taking into account Remark 2.5 we get a contradiction.

Suppose then that $\partial M$ lies strictly above $h(\Delta)$. Then there exists $\theta \in]-\frac{\pi}{4}, 0[$ sufficiently small and $f$ an hyperbolic rotation in $\mathbb{L}^{3}$ such that $f(\Pi)=\Pi_{\theta}$ and $f(\partial M)$ remains strictly above $h(\Delta)$. Note that the tangent plane to the maximal surface $f(M)$ at $(0,0,0)$ is $\Pi_{\theta}$ and thus we can assert that there are points of $f(M)$ below $h(\Delta)$. Translating in the positive $x_{3}$-direction, we find a last contact point with $h(\Delta)$ which must be an interior regular point. As in the previous case, by using the maximum principle we obtain that $h(\Delta)$ coincides with the translate of $f(M)$ by some vector $\left(0,0, t_{0}\right)$, for $t_{0}>0$. From Lemma 2.6 we see that $\pi^{-1}(0,0,0)$ intersects this translate at $\left(0,0, t_{0}\right)$. But this implies $\left(0,0, t_{0}\right)=(0,0,0)$, again a contradiction.

Proposition 2.10. Let $M$ be a compact maximal surface with isolated singularities. Then $M$ lies in the convex hull of $\partial M$ and its general conelike singularities.

Proof. Let $A$ be the set of general conelike singularities. If $M$ is contained in a plane the result is obvious. Assume $M$ is not flat and consider $v \in \mathbb{S}^{2}$ and $y \in \mathbb{R}^{3}$ such that $(\partial(M) \cup A) \subset H^{+}(y, v)$. We prove that $M \subset H^{+}(y, v)$.

We proceed by contradiction. Suppose that $M \cap\left(H^{-}(y, v)-H(y, v)\right) \neq \varnothing$. Let $M^{\prime}$ be a connected component of $M \cap H^{-}(y, v)$; then $M^{\prime}$ does not contain general conelike singularities.

First, assume $v$ is a timelike vector, that is, $H(y, v)$ is a spacelike plane. There exists an interior point $p \in M^{\prime}$ such that $M^{\prime}$ is contained in the slab determined by the parallel planes $H(p, v)$ and $H(y, v)$. Therefore we can use Proposition 2.9 to infer that $p$ is a regular point of $M^{\prime}$. Using the maximum principle we find $M^{\prime}=H(p, v)$, a contradiction. 
Analogously, if $v$ is either spacelike or lightlike, we can deduce the existence of an interior point $p \in M^{\prime}$ such that $M^{\prime}$ is contained in the slab determined by the parallel planes $H(p, v)$ and $H(y, v)$. If $p$ were a spacelike singularity, Lemma 2.4 implies $H(p, v)$ is the tangent plane to $M^{\prime}$ at $p$, contradicting $|g(q)|<1$. Assume $p$ is a lightlike singularity. Up to a Lorentzian isometry we can assume that $p=(0,0,0)$ and

- $H(p, v)=\Sigma_{\theta}$ and $M^{\prime} \subset \Sigma_{\alpha}^{-}$if $v$ is spacelike,

- $H(p, v)=\Pi_{\pi / 4}$ and $M^{\prime} \subset \Pi_{\pi / 4}^{+}$if $v$ is lightlike.

By Lemma 2.6, $M^{\prime}$ is in the exterior of $C((0,0,0))$. Consider $\pi$, the Lorentzian orthogonal projection onto $\Pi_{0}$. It is easy to prove that the preceding conditions imply that $\pi\left(M^{\prime}\right) \subset\left(\Pi_{0}-\{(0, y, 0) \mid y \in \mathbb{R}\}\right)$ in a neighborhood of $(0,0,0)$. This contradicts Lemma 2.1. Therefore, since $p$ is not a singular point we infer that $H(y, v)$ is the tangent plane to $M^{\prime}$ at $p$, in contradiction with the fact that $M$ is spacelike.

Remark 2.11. Proposition 2.10 holds even if $M$ cannot be extended to an open maximal surface $M^{\prime}$.

\section{Maximal surfaces whose boundary is contained in a timelike plane}

Recall that we defined

$$
\mathrm{C}^{+}=\left\{\left(x_{1}, x_{2}, x_{3}\right) \in \mathbb{R}^{3} \mid x_{1}^{2}+x_{2}^{2}-x_{3}^{2} \leq 0, x_{3} \geq 0\right\} .
$$

For $\theta \in] 0, \frac{\pi}{4}\left[\right.$ and $\left.\delta, \delta^{\prime} \in\right] 0, \pi[$, we also define the convex region

$$
V\left(\theta, \delta, \delta^{\prime}\right)=\Pi_{0}^{+} \cap \Pi_{\theta}^{-} \cap H^{+}\left((0,0,0),\left(1,-\cot \delta, \cot \theta\left(\cot \delta-\cot \delta^{\prime}\right)\right)\right) .
$$

This is the convex hull of the half-lines with origin in $(0,0,0)$ and directions $(1,0,0),(\cot \delta, 1,0)$ and $\left(\cot \delta^{\prime}, 1, \tan \theta\right)$ (see figure).

Let $\tau_{t}$ denote the translation along the vector $(0,0, t)$, where $t \in \mathbb{R}$.

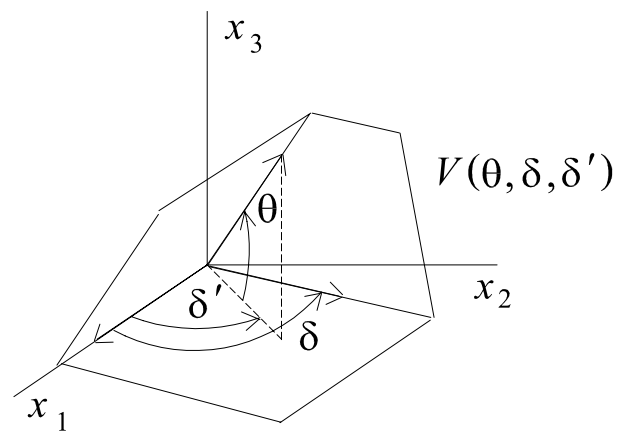


Proposition 3.1. Let $\alpha \in]-\frac{\pi}{4}, \frac{\pi}{4}$ [ be arbitrary. Suppose $M$ is a connected, properly immersed maximal surface contained in $\Pi_{\pi / 4}^{+} \cap \Sigma_{\alpha}^{+}$, such that $\partial M \subset \Sigma_{\alpha}$. If there is a point $p_{0} \in \partial M$ minimizing $x_{3}$ on $\partial M$, there must be some downward pointing lightlike singularity in the interior of $M$.

Proof. After applying an orthochronous hyperbolic rotation $f_{s}$ of $\mathbb{L}^{3}$, we can assume $\alpha=0$.

Let $M$ be as in the hypothesis of the proposition and define $\hat{t}=x_{3}\left(p_{0}\right)$. For any $\theta \in\left[0, \frac{\pi}{4}[\right.$, consider the set

$$
\Phi_{\theta}=\left\{t \in[0, \hat{t}] \mid M \subset \Pi_{\theta t}^{+}\right\} .
$$

Since $\Phi_{\theta}$ contains 0 , it is nonempty. Suppose there is no singularity in Int $M$ as in the conclusion; we shall prove that $\Phi_{\theta}=[0, \hat{t}]$, and from there we will derive a contradiction.

We may assume $\hat{t}>0$, the case $\hat{t}=0$ being obvious. Clearly $I_{\theta}$ is closed; we show that it is open. If $t \in \Phi_{\theta}$ then $[0, t] \subset \Phi_{\theta}$. We claim that if $t \in \Phi_{\theta} \cap[0, \hat{t}[$, there exists $\varepsilon>0$ such that $\left[t, t+\varepsilon\left[\subset \Im_{\theta}\right.\right.$. If not, we have two possibilities: either

(a) there is an interior point $p$ of $M$ in the plane $\Pi_{\theta t}$, or

(b) $M$ is asymptotic to $\Pi_{\theta t}$ at infinity.

In case (a), $p$ is not a singularity, for if it were, it would be downward pointing by Proposition 2.9(i), contrary to assumption. But if $p$ is not a singularity, the interior maximum principle implies that $M$ and $\Pi_{\theta t}$ coincide, in contradiction with the inequality $x_{3}\left(p_{0}\right)=\hat{t}>t$.

In case (b), we can assume $M \cap \Pi_{\theta t}=\varnothing$; otherwise there exists an interior point of $M$ in $\Pi_{\theta t}$ and we may apply the previous argument. Consider an orthochronous isometry $f$ of $\mathbb{\complement}^{3}$ such that $f\left(\Pi_{\theta t}\right)=\Pi_{0}$ and $f\left(\Pi_{\theta t}^{+}\right)=\Pi_{0}^{+}$. Set $\tilde{M}=f(M)$. Then we have a properly immersed maximal surface $\widetilde{M} \subset \Pi_{0}^{+}$asymptotic to $\Pi_{0}$ and disjoint from it.

Since the immersion is proper and $(0,0,0) \notin \widetilde{M}$ we can find $\epsilon>0$ sufficiently small so that the ball $B(\epsilon)$ of radius $\epsilon$ around $(0,0,0)$ is disjoint from $\tilde{M}$. Hence, there exists $\left.\epsilon^{\prime} \in\right] 0, \epsilon\left[\right.$ and $a_{0}>0$ small enough such that $\tau_{\epsilon^{\prime}}\left(C_{a_{0}}\right) \subset B(\epsilon) \cup \Pi_{0}^{-}$. Now define

$$
\left.\left.A=\{a \in] 0, a_{0}\right] \mid \tau_{\epsilon^{\prime}}\left(C_{a}\right) \cap \tilde{M}=\varnothing\right\} .
$$

Clearly, $a_{0} \in A$ and we can consider the infimum $a^{\prime}$ of $A$. We claim that $a^{\prime}=0$. Assume on the contrary that $a^{\prime}>0$. Since $\tau_{\epsilon^{\prime}}\left(C_{a}\right)$ and $\tilde{M}$ do not have a contact at infinity, there exists an interior point $p$ of $\widetilde{M}$ in $\tau_{\epsilon^{\prime}}\left(C_{a^{\prime}}\right)$. Taking into account Proposition 2.9(i) and the assumed absence of singularities, we see that $p$ is a regular point of $\widetilde{M}$. Applying the maximum principle, we obtain $\widetilde{M}=\tau_{\epsilon^{\prime}}\left(C_{a^{\prime}}\right)$, in contradiction with $\partial \widetilde{M} \subset \Sigma_{0} \cap \Pi_{0}^{+}$. 
We have shown that if the conclusion of the theorem fails, then $\Phi_{\theta}$ is open and $\Phi_{\theta}=[0, \hat{t}]$. Hence $M \subset \Pi_{\theta \hat{t}}^{+}$for all $\theta \in\left[0, \frac{\pi}{4}\left[\right.\right.$ and thus $M \subset \Pi_{\pi / 4, \hat{t}}^{+}$. Now consider $p_{0}$ : if it is not a singular point in $\partial M$, the fact that $p_{0} \in \partial(M) \cap \Pi_{\pi / 4, \hat{t}}$ and $M \subset \Pi_{\pi / 4, \hat{t}}^{+} \cap \Sigma_{0}^{+}$implies that the tangent plane $p_{0}$ is lightlike of timelike, which is a contradiction. If instead $p_{0}$ is a singular point, Lemma 2.6 implies that around $p_{0}$ the surface is in the exterior of $C\left(p_{0}\right)$, which again contradicts $M \subset \Pi_{\pi / 4, \hat{t}}^{+}$.

Corollary 3.2. There exists no connected properly immersed maximal surface $M$ without downward pointing lightlike singularities in the interior and such that $M \subset$ $\mathrm{C}^{+} \cap \Sigma_{\alpha}^{+}$and $\partial M \subset \Sigma_{\alpha}$, for any $\left.\alpha \in\right]-\frac{\pi}{4}, \frac{\pi}{4}[$.

Proof. This follows immediately from Proposition 3.1.

Corollary 3.3. There exists no connected properly immersed maximal surface $M$ without downward pointing lightlike singularities in the interior and such that $M \subset$ $\mathrm{C}^{+}$and $\mathrm{\partial} M$ lies in the intersection of $\mathrm{C}^{+}$with a timelike plane $P$.

Proof. Assume that there exists such a maximal surface and consider a connected component $M^{\prime}$ of $M-(M \cap P)$. Up to an elliptic rotation and a translation we can assume the timelike plane is the plane $\Sigma_{\alpha}$, for $\left.\alpha \in\right]-\frac{\pi}{4}, \frac{\pi}{4}\left[, M^{\prime} \subset \mathrm{C}^{+} \cap \Sigma_{\alpha}^{+}\right.$and $\partial M^{\prime} \subset \Sigma_{\alpha}$. An immediate application of Corollary 3.2 to $M^{\prime}$ leads to a contradiction.

Theorem 3.4. There exists no connected properly immersed maximal surface $M$ without downward pointing lightlike singularities in the interior and such that $M \subset$ $W_{\delta} \cap \Sigma_{\alpha}^{+}$and $\partial M \subset \Sigma_{\alpha} \cap C^{+}$, for $\left.\delta \in\right] 0, \frac{\pi}{4}[, \alpha \in]-\frac{\pi}{4}, \frac{\pi}{4}[$.

Proof. Consider the isometry $f_{\varepsilon}$ of $\mathbb{L}^{3}$ with $\tanh \varepsilon=\tan \frac{\pi}{8}$. It is not difficult to see that $f_{\varepsilon}(M) \subset \Pi_{\pi / 8}^{+} \cap W_{\delta^{\prime}} \cap \Sigma_{\alpha^{\prime}}^{+}$and $\partial f_{\varepsilon}(M) \subset \Sigma_{\alpha^{\prime}} \cap C^{+}$, where

$$
\begin{gathered}
\tan \alpha^{\prime}=\frac{\tan \alpha+\tan \frac{\pi}{8}}{\tan \frac{\pi}{8} \tan \alpha+1}, \\
\tan \delta^{\prime}=\min \left\{\tan \frac{\pi}{8}, \cosh \varepsilon \tan \delta\left(\tan \frac{\pi}{8} \tan \alpha+1\right)\right\} .
\end{gathered}
$$

For simplicity of notation we consider $M \subset \Pi_{\pi / 8}^{+} \cap W_{\delta} \cap \Sigma_{\alpha}^{+}$and $\partial M \subset \Sigma_{\alpha} \cap C^{+}$. We claim that $(0,0,0) \notin \partial M$ and so $\partial M \subset \Pi_{0}^{+}-\Pi_{0}$. If not, we deduce from Lemma 2.6 that around $(0,0,0)$ the maximal surface $M$ is in the exterior of $C((0,0,0))$, but this contradicts $\partial M \subset \mathrm{C}^{+}$.

Now consider the Scherk-type maximal surface $S_{\delta / 2}$ (page 13) asymptotic to the boundary of the region $W_{\delta / 2}$. We will prove that $S_{\delta / 2} \cap M=\varnothing$. It is clear that there exist $\left.\left.t_{0} \in\right]-\infty, 0\right]$ and $\left.t_{1} \in\right] 0, \infty\left[\right.$ such that $\tau_{t_{0}}\left(S_{\delta / 2}\right) \cap M=\varnothing$ and $\tau_{t_{1}}\left(S_{\delta / 2}\right) \cap M \neq \varnothing$. Therefore, we can define

$$
\hat{t}=\operatorname{infimum}\{t \in] t_{0}, \infty\left[\mid \tau_{t}\left(S_{\delta / 2}\right) \cap M \neq \varnothing\right\} .
$$


Suppose $\hat{t} \leq 0$. Observe that, since $M \subset \Pi_{\pi / 8}^{+} \cap W_{\delta} \cap \Sigma_{\alpha}^{+}, \partial M \subset C^{+}-\{(0,0,0)\}$ and $S_{\delta / 2} \cap C^{+}=\{(0,0,0)\}$, then $\tau_{\hat{t}}\left(S_{\delta / 2}\right)$ and $M$ can have a contact point neither at infinity nor at the boundary. Hence there exists an interior point of $M$ in $\tau_{\hat{t}}\left(S_{\delta / 2}\right)$. Taking into account our assumptions on the singularities and Proposition 2.9(i) we deduce that this point is not a singularity. Then, by applying the maximum principle we get that $M$ and $\tau_{\hat{t}}\left(S_{\delta / 2}\right)$ coincide which contradicts the hypothesis on $\partial M$. Thus $\hat{t}>0$ and $S_{\delta / 2} \cap M=\varnothing$.

Now consider $S_{\delta / 2}^{\lambda}$, the homothetic shrinking of $S_{\delta / 2}$ by $\lambda, \lambda \geq 1$. We shall prove that $S_{\delta / 2}^{\lambda} \cap M=\varnothing$ for all $\lambda \geq 1$. Suppose on the contrary that there exists $\lambda^{\prime} \geq 1$ such that $S_{\delta / 2}^{\lambda^{\prime}} \cap M \neq \varnothing$. We set

$$
\hat{\lambda}=\operatorname{infimum}\{\lambda \in] 1, \infty\left[\mid S_{\delta / 2}^{\lambda} \cap M \neq \varnothing\right\} .
$$

Clearly $S_{\delta / 2}^{\hat{\lambda}}$ and $M$ do not touch either at infinity or at the boundary. Therefore there must exist an interior point of $M$ in $S_{\delta / 2}^{\hat{\lambda}}$. Again using Proposition 2.9(i) and our hypothesis on the singularities we deduce that this point is not a singularity and so by applying the maximum principle we obtain that $S_{\delta / 2}^{\hat{\lambda}}$ and $M$ coincide. But this contradicts our assumptions on $\partial M$.

Thus $S_{\delta / 2}^{\lambda} \cap M=\varnothing$ for all $\lambda \geq 1$. Taking into account that $S_{\delta / 2}$ is asymptotic to $C^{+}((0,0,0))$ near the conelike singularity $(0,0,0)$, we deduce that $M \subset \mathrm{C}^{+}$and the Corollary 3.2 finishes the proof.

Theorem 3.5. There exist no connected properly immersed maximal surface $M$ without downward pointing lightlike singularities in the interior and such that $M \subset$ $\Pi_{\theta}^{+} \cap \Sigma_{\alpha}^{+}$and $\partial M \subset \Sigma_{\alpha} \cap C^{+}$, for $\left.\theta, \alpha \in\right]-\frac{\pi}{4}, \frac{\pi}{4}[$.

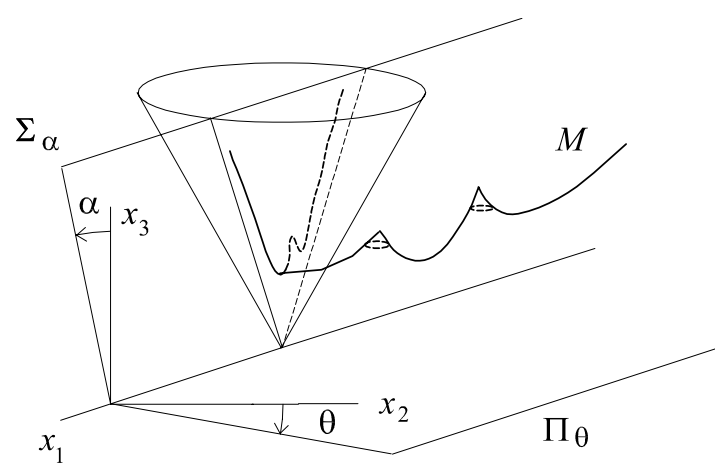

Proof. Suppose there exists such an $M$. We observe that if $\theta \leq 0$ or $\alpha<0$ we can consider an orthochronous hyperbolic rotation $f_{s}$ such that $f_{s}(M) \subset \Pi_{\theta^{\prime}}^{+} \cap \Sigma_{\alpha^{\prime}}^{+}$and $\partial f_{s}(M) \subset \Sigma_{\alpha^{\prime}} \cap C^{+}$for some $\left.\theta^{\prime} \in\right] 0, \frac{\pi}{4}\left[, \alpha^{\prime} \in\left[0, \frac{\pi}{4}[\right.\right.$. As in the previous theorem, for the sake of simplicity of notation we assume $M \subset \Pi_{\theta}^{+} \cap \Sigma_{\alpha}^{+}$and $\partial M \subset \Sigma_{\alpha} \cap C^{+}$, for $\theta \in] 0, \frac{\pi}{4}\left[, \alpha \in\left[0, \frac{\pi}{4}[\right.\right.$. 
Since $\partial M \subset \Sigma_{\alpha} \cap C^{+}$we have that there exists $p_{0} \in \partial M$ such that $x_{3}\left(p_{0}\right) \leq x_{3}(p)$ for all $p \in \partial M$. As in the preceding theorem it is easy to see that $p_{0} \neq(0,0,0)$ and so $\lambda=x_{3}\left(p_{0}\right)>0$. Then, reasoning as in Proposition 3.1 we can conclude $M \subset \Pi_{\theta \lambda}^{+} \cap \Sigma_{\alpha}^{+}$.

Denote by $\widetilde{R}_{\delta}$ the Riemann type maximal example that results after applying an elliptic rotation of $\frac{\pi}{2}$ along the axis $x_{3}$ on $R_{\delta}$ for any $\left.\delta \in\right] 0, \frac{\pi}{4}$ [. We assert that $M \cap \widetilde{R}_{\delta}=\varnothing$. Observe that we can consider $t_{0} \leq 0$ and $t_{1} \in \mathbb{R}$ such that $\tau_{t_{0}}\left(\widetilde{R}_{\delta}\right) \cap M=\varnothing$ and $\tau_{t_{1}}\left(\widetilde{R}_{\delta}\right) \cap M \neq \varnothing$. Now define

$$
\hat{t}=\operatorname{infimum}\{t \in] t_{0}, \infty\left[\mid \tau_{t}\left(\widetilde{R}_{\delta}\right) \cap M \neq \varnothing\right\} .
$$

Suppose $\hat{t} \leq 0$. Note that $\tau_{\hat{t}}\left(\widetilde{R}_{\delta}\right)$ and $M$ can have a contact point neither at infinity nor at the boundary. Hence there exists an interior point of $M$ in $\tau_{\hat{t}}\left(\widetilde{R}_{\delta}\right)$. Making use of Proposition 2.9(i) and taking into account our assumptions on singularities, we deduce that the point is not a singularity. Therefore, by applying the maximum principle we get that $\tau_{\hat{t}}\left(\widetilde{R}_{\delta}\right)$ and $M$ coincide. But this contradicts our hypothesis on $\partial M$. Thus $\hat{t}>0$ and $\widetilde{R}_{\delta} \cap M=\varnothing$.

Consider now $\widetilde{R}_{\delta}^{\lambda}$ the homothetic shrinking of $\widetilde{R}_{\delta}$ by $\lambda, \lambda>0$. Next we prove that $\widetilde{R}_{\delta}^{\lambda} \cap M=\varnothing$ for all $\lambda \geq 1$. Assume that there exists $\lambda^{\prime}>1$ such that $\widetilde{R}_{\delta}^{\lambda^{\prime}} \cap M \neq \varnothing$. We define

$$
\hat{\lambda}=\operatorname{infimum}\{\lambda \in] 1, \lambda^{\prime}\left[\mid \widetilde{R}_{\delta}^{\lambda} \cap M \neq \varnothing\right\} .
$$

Observe that $\widetilde{R}_{\delta}^{\lambda^{\prime}}$ and $M$ do not touch either at infinity or at the boundary for all $\lambda \geq 1$. Therefore there is an interior point of $M$ in $\widetilde{R}_{\delta}^{\lambda^{\prime}}$. Using again our assumptions on singularities and Proposition 2.9(i) we deduce that the point is not a singularity. Then by applying the maximum principle we obtain that $\widetilde{R}_{\delta}^{\lambda^{\prime}}$ and $M$ coincide, which contradicts our hypothesis on $\partial M$. The same argument proves that $\widetilde{R}_{\delta}^{\lambda} \cap M=\varnothing$ for all $\lambda \leq 1$.

Analogously, considering $\widehat{R}_{\delta}$ the Riemann type maximal example that results after applying a rotation of $-\frac{\pi}{2}$ along the axis $x_{3}$ on $R_{\delta}$ for any $\left.\delta \in\right] 0, \frac{\pi}{4}$ [, we obtain $\widehat{R}_{\delta}^{\lambda} \cap M=\varnothing$ for all $\lambda \in \mathbb{R}$.

Furthermore, it is not difficult to prove that

$$
\begin{array}{r}
\left(\Pi_{\theta}^{+} \cap \Sigma_{\alpha}^{+}\right)-\left(\bigcup_{\lambda \in \mathbb{R}} \widetilde{R}_{\delta}^{\lambda} \cup \bigcup_{\lambda \in \mathbb{R}} \widehat{R}_{\delta}^{\lambda}\right) \subset\left\{\left(x_{1}, x_{2}, x_{3}\right) \in \mathbb{R}^{3} \mid-\tan \delta x_{1}+x_{2}+x_{3} \geq 0\right\} \\
\cap\left\{\left(x_{1}, x_{2}, x_{3}\right) \in \mathbb{R}^{3} \mid \tan \delta x_{1}+x_{2}+x_{3} \geq 0\right\} .
\end{array}
$$

Taking this into account, we can assert $M \subset\left(\Pi_{\theta}^{+} \cap \Sigma_{\alpha}^{+}\right) \cap\left\{\left(x_{1}, x_{2}, x_{3}\right) \in \mathbb{R}^{3} \mid-\tan \delta x_{1}+x_{2}+x_{3} \geq 0, \tan \delta x_{1}+x_{2}+x_{3} \geq 0\right\}$. A direct computation shows that $\Pi_{\theta}^{+} \cap\left\{\left(x_{1}, x_{2}, x_{3}\right) \in \mathbb{R}^{3} \mid-\tan \delta x_{1}+x_{2}+x_{3} \geq 0, \tan \delta x_{1}+x_{2}+x_{3} \geq 0\right\} \subset W_{\delta^{\prime}}$, 
where $\left.\delta^{\prime} \in\right] 0, \frac{\pi}{4}\left[\right.$ is given by $\tan \delta^{\prime}=\tan \delta \tan \theta /(1+\tan \theta)$. Then Theorem 3.4 concludes the proof.

To finish this section, we analyze the case of maximal surfaces whose boundary is contained in a timelike plane but not necessarily in $\mathrm{C}^{+}$.

Proposition 3.6. There exists no connected properly immersed maximal surface $M$ with at least one connected component of $\partial M$ contained in the intersection $\Sigma_{\alpha}^{+} \cap \Sigma_{-\alpha}^{+} \cap\left\{\left(x_{1}, x_{2}, x_{3}\right) \in \mathbb{R}^{3} \mid x_{1}=0\right\}$, where $\left.\alpha \in\right] 0, \frac{\pi}{4}[$.

Proof. Let $B$ a connected component of $\partial M$ satisfying

$$
B \subset \Sigma_{\alpha}^{+} \cap \Sigma_{-\alpha}^{+} \cap\left\{\left(x_{1}, x_{2}, x_{3}\right) \in \mathbb{R}^{3} \mid x_{1}=0\right\},
$$

where $\alpha \in] 0, \frac{\pi}{4}\left[\right.$. The function $x_{2}$ has at least one minimum on $B$, and this minimum cannot be a singularity. Then, the tangent vector to the boundary at this point is vertical and therefore the tangent plane of the maximal surface at this point is timelike, which is contrary to our assumptions.

Corollary 3.7. There exists no connected properly immersed maximal surface $M$ contained in $V\left(\theta, \delta, \delta^{\prime}\right)$ with $\partial M$ contained in a timelike plane.

Proof. From the hypothesis it is not difficult to see that there exists an isometry of $\mathbb{L}^{3}$ that sends the timelike plane to the plane $\left\{\left(x_{1}, x_{2}, x_{3}\right) \in \mathbb{R}^{3} \mid x_{1}=0\right\}$ and in particular the image of $\partial M$ lies in $\Sigma_{\alpha}^{+} \cap \Sigma_{-\alpha}^{+} \cap\left\{\left(x_{1}, x_{2}, x_{3}\right) \in \mathbb{R}^{3} \mid x_{1}=0\right\}$ for some $\alpha \in] 0, \frac{\pi}{4}[$. The result is then a consequence of Proposition 3.6.

\section{Maximal surfaces whose boundary is contained in a spacelike plane}

We now obtain, using the maximum principle, other results about maximal surfaces whose boundary is contained in a spacelike plane but that cannot be inferred from the theorem of Fernández and López quoted on page 9. We start with a result similar to Corollary 2.8.

Proposition 4.1. Let $M$ be a connected properly immersed maximal surface without downward pointing lightlike singularities in the interior such that

$$
M \subset\left\{\left(x_{1}, x_{2}, x_{3}\right) \in \mathbb{R}^{3} \mid 0 \leq x_{3} \leq k\right\} \quad \text { and } \quad \partial M \subset\left\{\left(x_{1}, x_{2}, x_{3}\right) \in \mathbb{R}^{3} \mid x_{3}=k\right\},
$$

for $k>0$. Then $M$ is a planar region.

Proof. We proceed by contradiction. Assume that there exists $t \geq 0$ such that $M \subset \Pi_{0 t}^{+}$but $M \not \subset \Pi_{0 t^{\prime}}^{+}$for any $t<t^{\prime}$. We claim that $M \cap \Pi_{0 t}=\varnothing$. If not, there exists an interior point $p$ of $M$ in $\Pi_{0 t}$. Then, from Proposition 2.9(i) and our assumptions on singularities, we deduce that $p$ is a regular point. It then follows from the maximum principle that $M=\Pi_{0 t}$, contradicting the hypothesis on $\partial M$. 


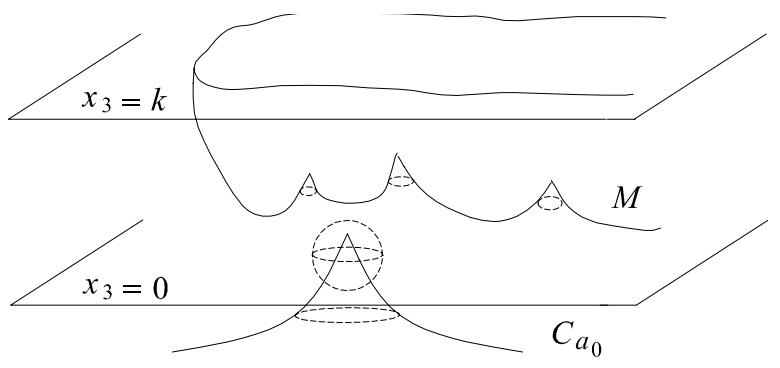

Since $M$ is properly immersed and $M \cap \Pi_{0 t}=\varnothing$ we can find $\varepsilon>0$ such that the ball of radius $\varepsilon$ about $(0,0, t)$ does not meet $M$. Hence, there are constants $\left.\varepsilon^{\prime} \in\right] 0, \varepsilon\left[\right.$ and $a_{0}>0$ sufficiently small such that $\tau_{t+\varepsilon^{\prime}}\left(C_{a_{0}}\right)$ is contained in $\Pi_{0 t}^{-}$ and also in the ball of radius $\varepsilon$ around $(0,0, t)$. Now define

$$
\left.\left.A=\{a \in] 0, a_{0}\right] \mid \tau_{t+\varepsilon^{\prime}}\left(C_{a}\right) \cap M=\varnothing\right\} .
$$

Clearly, $a_{0} \in A$ and we can consider the infimum $a^{\prime}$ of $A$. We claim $a^{\prime}=0$. Assume on the contrary that $a^{\prime}>0$. Then as $\tau_{t+\varepsilon^{\prime}}\left(C_{a}\right)$ and $M$ do not have a contact either at infinity or at the boundary, we infer that there is an interior point of $M$ in $\tau_{t+\varepsilon^{\prime}}\left(C_{a^{\prime}}\right)$. Taking into account Proposition 2.9(i) and our assumptions on the singularities we infer that the interior point is not a singularity and then, by applying the interior maximum principle we obtain $M=\tau_{t+\varepsilon^{\prime}}\left(C_{a^{\prime}}\right)$ which contradicts the hypothesis on $\partial M$.

Therefore, $a^{\prime}=0$ and so $M \subset \Pi_{0 t+\varepsilon^{\prime}}^{+}$contradicting our assumption at the beginning of the proof.

Theorem 4.2. Let $M$ be a connected properly immersed maximal surface without upward pointing lightlike singularities in the interior such that $M \subset V\left(\theta, \delta, \delta^{\prime}\right)$ and $\partial M \subset \Pi_{0}$. Then $M$ is a planar region.

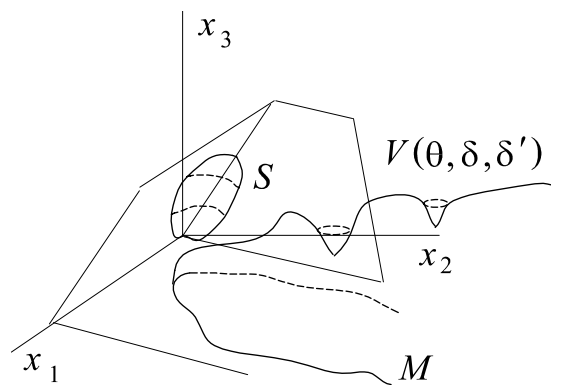

Proof. Up a translation we can assume that

$$
M \cap\left(\Pi_{\theta} \cup H\left((0,0,0),\left(1,-\cot \delta, \cot \theta\left(\cot \delta-\cot \delta^{\prime}\right)\right)\right)=\varnothing .\right.
$$


Now we observe that

$$
\begin{gathered}
\tau_{-1}\left(\mathbb{R}_{+}^{2}\right) \cap \Pi_{\theta} \cap V\left(\theta, \delta, \delta^{\prime}\right)=\alpha_{1}, \\
\tau_{-1}\left(\mathbb{T}_{+}^{2}\right) \cap H\left((0,0,0),\left(1,-\cot \delta, \cot \theta\left(\cot \delta-\cot \delta^{\prime}\right)\right)\right) \cap V\left(\theta, \delta, \delta^{\prime}\right)=\alpha_{2},
\end{gathered}
$$

where $\alpha_{1}$ and $\alpha_{2}$ are regular curves. The union of these curves is a continuous curve of $\tau_{-1}\left(\mathbb{M}_{+}^{2}\right)$ and the tangent vectors to these curves at the point $(0,0,0)$ are contained in the plane $\Pi_{0}$ and are linearly independent.

Since $\tau_{-1}\left(\mathbb{H}_{+}^{2}\right)$ is a spacelike surface, it is well-known (see Theorem 4.1 in [Bartnik and Simon 1982/83]) that there exists $S$ a maximal surface (it is even a graph on the $x_{3}$-plane) spanned by the curve $\alpha_{1} \cup \alpha_{2}$. Note that the tangent plane of $S$ at $(0,0,0)$ is the plane $\Pi_{0}$. On the other hand, using Proposition 2.10 and Remark 2.11, we see that $S$ is contained in the convex hull of its boundary and thus $S \subset V\left(\theta, \delta, \delta^{\prime}\right)$.

Now, we denote by $S^{\lambda}$ the homothetic shrinking of $S$ by $\lambda, \lambda>0$. As $M$ is properly immersed it is possible to find $\lambda_{0}>0$ such that $S^{\lambda_{0}} \cap M=\varnothing$. Next we prove that $S^{\lambda} \cap M=\varnothing$ for all $\lambda>0$. Assume that there exists $\lambda^{\prime}>0$ such that $S^{\lambda^{\prime}} \cap M \neq \varnothing$. We denote by

$$
\left.\left.\hat{\lambda}=\operatorname{infimum}\{\lambda \in] \lambda_{0}, \lambda^{\prime}\right] \mid S^{\lambda} \cap M \neq \varnothing\right\} .
$$

Observe that $S^{\lambda}$ and $M$ do not contact at the boundary for all $\lambda>0$. Therefore there is an interior point of $M$ in $S^{\hat{\lambda}}$. It follows from Proposition 2.9(ii) and the conditions on the singularities that the contact point is not a singularity. Then, by applying the maximum principle we obtain $S^{\lambda}=M$ which contradicts the assumptions on $\partial M$.

Hence, taking into account that the tangent plane of $S$ at $(0,0,0)$ is $\Pi_{0}$ we obtain

$$
V\left(\theta, \delta, \delta^{\prime}\right)-\bigcup_{\lambda \in \mathbb{R}} S^{\lambda} \subset \Pi_{0},
$$

from which we deduce that $M \subset \Pi_{0}$.

Corollary 4.3. Let $M$ be a connected properly immersed maximal surface without general conelike singularities in the interior such that $M \subset V\left(\theta, \delta, \delta^{\prime}\right)$ and $\partial M$ is contained in a spacelike plane. Then $M$ is a planar region.

Proof. Let $\Pi$ be the spacelike plane such that $\partial M \subset \Pi$ and $M^{\prime}$ a connected component of $M-(M \cap \Pi)$. Denote by $\Pi^{+}$the half-space determined by $\Pi$ such that $M^{\prime} \subset \Pi^{+}$. Then, it is not difficult to see that there exists an isometry of $\mathbb{L}^{3}, \mathrm{f}$, that verifies $f(\Pi)=\Pi_{0}$ and $f\left(V\left(\theta, \delta, \delta^{\prime}\right) \cap \Pi^{+}\right) \subset V\left(\hat{\theta}, \hat{\delta}, \hat{\delta}^{\prime}\right)$, for some $\hat{\theta}, \hat{\delta}$ and $\hat{\delta}^{\prime}$. Therefore, the corollary follows from Theorem 4.2.

By contrast: 
Proposition 4.4. Let $M$ be a connected properly immersed maximal surface without general conelike singularities in the interior such that $M \subset \mathrm{C}^{+}$and $\partial \mathrm{M}$ is contained in a spacelike plane. Then $M$ is a planar region.

Proof. Our hypotheses imply that $\partial M$ is compact. We consider the intersection of $M$ with all the timelike planes $H(y, v)$ such that $\partial M \subset H^{+}(y, v)$. By applying Corollary 3.3 to the connected components of $M$ contained in $H^{-}(y, v)$ we obtain that $M \subset H^{+}(y, v)$ for all the timelike planes described above. Then $M$ is also compact and Proposition 2.10 proves that $M$ is a planar region.

\section{Maximal surfaces whose boundary is contained in a lightlike plane and the convex hull property}

As a consequence of the previous sections we deduce the following results for maximal surfaces whose boundary is contained in a lightlike plane.

Proposition 5.1. There exists no connected properly immersed maximal surface $M$ without general conelike singularities in the interior and such that $M \subset V\left(\theta, \delta, \delta^{\prime}\right)$ and $\partial M$ is contained in a lightlike plane.

Proof. Suppose there exists such an $M$. Let $\Pi$ be the lightlike plane such that $\partial M \subset \Pi$. Then, we can consider the pencil of planes through the line $L=\Pi \cap \Pi_{0}$, that is, the set of planes sharing the line $L$. Since $M$ cannot be flat, it is possible to find a spacelike or timelike plane in the pencil that intersects $M$ transversally. But Corollaries 4.3 and 3.7 lead to a contradiction in each case.

Proposition 5.2. There exists no connected properly immersed maximal surface $M$ without general conelike singularities in the interior and such that $M \subset \mathrm{C}^{+}$and $\partial M$ is contained in a lightlike plane.

Proof. This can be demonstrated like Proposition 5.1, but using Proposition 4.4 and Corollary 3.3.

As we saw in Section 2, a compact maximal surface lies in the convex hull of its boundary and the set of its general conelike singularities. This is not true for noncompact maximal surfaces in general. However, Theorem 4.2 and Proposition 4.4 can be seen as a convex hull type property. We have proved that if certain conditions are satisfied then the surfaces lie in the convex hull of their boundary. In the remainder of the section, we use the results obtained in the previous sections to give a generalization of these results. More precisely:

Proposition 5.3. Any connected properly immersed maximal surface contained in $V\left(\theta, \delta, \delta^{\prime}\right)$ lies in the convex hull of its boundary and its general conelike singularities. 
Proof. Let $M$ be a minimal surface satisfying the hypotheses of the proposition and denote by $A$ the set of general conelike singularities of $M$. If $M$ is contained in a plane the result is obvious. Assume then that $M$ is not flat and consider $v \in \mathbb{S}^{2}$ and $y \in \mathbb{R}^{3}$ such that $(\partial(M) \cup A) \subset H^{+}(y, v)$. We have to prove that $M \subset H^{+}(y, v)$ too.

We proceed by contradiction, and suppose that $M \cap\left(H^{-}(y, v)-H(y, v)\right) \neq \varnothing$. Let $M^{\prime}$ be a connected component of $M \cap H^{-}(y, v)$.

If $v$ is spacelike, so $H(y, v)$ is a timelike plane, Corollary 3.7 leads to a contradiction.

If $v$ is timelike, so $H(y, v)$ is spacelike, the assumption that $M^{\prime}$ is not flat contradicts Corollary 4.3.

Finally, if $v$ is lightlike, so is the plane $H(y, v)$, and Proposition 5.1 gives a contradiction.

Proposition 5.4. Any connected properly immersed maximal surface contained in $\mathrm{C}^{+}$lies in the convex hull of its boundary and its general conelike singularities.

Proof. The proof is obtained as for the preceding proposition, using Corollary 3.3 and Propositions 5.2 and 4.4.

\section{Acknowledgments}

We are indebted to F. Martín, I. Fernández and especially to F. J. López for helpful conversations during the preparation of this work. This paper was carried out during Ferrer's visit to IME-USP, Brazil. She is grateful to the people at the Institute for their hospitality. We also thank the referee for valuable suggestions.

\section{References}

[Bartnik and Simon 1982/83] R. Bartnik and L. Simon, "Spacelike hypersurfaces with prescribed boundary values and mean curvature", Comm. Math. Phys. 87:1 (1982/83), 131-152. MR 84j:58126 Zbl 0512.53055

[Calabi 1970] E. Calabi, "Examples of Bernstein problems for some nonlinear equations", pp. 223230 in Global analysis (Berkeley, 1968), vol. 2, edited by S.-S. Chern and S. Smale, Proc. Sympos. Pure Math. 15, Amer. Math. Soc., Providence, RI, 1970. MR 41 \#8806 Zbl 0211.12801

[Cheng and Yau 1976] S. Y. Cheng and S. T. Yau, "Maximal space-like hypersurfaces in the LorentzMinkowski spaces", Ann. of Math. (2) 104:3 (1976), 407-419. MR 55 \#4063 Zbl 0352.53021

[Dierkes et al. 1992] U. Dierkes, S. Hildebrandt, A. Küster, and O. Wohlrab, Minimal surfaces, I: Boundary value problems, Grundlehren der Math. Wissenschaften 295, Springer, Berlin, 1992. MR 94c:49001a Zbl 0777.53012

[Ecker 1986] K. Ecker, "Area maximizing hypersurfaces in Minkowski space having an isolated singularity”, Manuscripta Math. 56:4 (1986), 375-397. MR 88b:53075 Zbl 0594.58023 
[Estudillo and Romero 1992] F. J. M. Estudillo and A. Romero, "Generalized maximal surfaces in Lorentz-Minkowski space L ${ }^{3}$ ", Math. Proc. Cambridge Philos. Soc. 111:3 (1992), 515-524. MR 93b:53010 Zbl 0824.53061

[Fernández and López 2004a] I. Fernández and F. J. López, "Periodic maximal surfaces in the Lorentz-Minkowski space [ [3", preprint, 2004. To appear in Math. Z. math.DG/0412461

[Fernández and López 2004b] I. Fernández and F. J. López, "Relative parabolicity of zero mean curvature surfaces in $\mathbb{R}^{3}$ and $\mathbb{R}_{1}^{3}$," preprint, 2004. math.DG/0410435

[Fernández et al. 2005] I. Fernández, F. J. López, and R. Souam, "The space of complete embedded maximal surfaces with isolated singularities in the 3-dimensional Lorentz-Minkowski space", Math. Ann. 332:3 (2005), 605-643. MR 2006h:58012 Zbl 1081.53052

[Gulliver et al. 1973] R. D. Gulliver, II, R. Osserman, and H. L. Royden, "A theory of branched immersions of surfaces", Amer. J. Math. 95 (1973), 750-812. MR 50 \#14595 Zbl 0295.53002

[Hoffman and Meeks 1990] D. Hoffman and W. H. Meeks, III, "The strong halfspace theorem for minimal surfaces”, Invent. Math. 101:2 (1990), 373-377. MR 92e:53010 Zbl 0722.53054

[Klyachin and Miklyukov 1993] A. A. Klyachin and V. M. Miklyukov, "Existence of solutions with singularities for the maximal surface equation in Minkowski space”, Mat. Sb. 184:9 (1993), 103124. In Russian; translated in Russian Acad. Sci. Sb. Math. 80 (1995), 87-104. MR 95j:53014 Zbl 0824.53062

[Kobayashi 1983] O. Kobayashi, "Maximal surfaces in the 3-dimensional Minkowski space $L^{3}$ ", Tokyo J. Math. 6:2 (1983), 297-309. MR 85d:53003 Zbl 0535.53052

[Kobayashi 1984] O. Kobayashi, "Maximal surfaces with conelike singularities", J. Math. Soc. Japan 36:4 (1984), 609-617. MR 86d:53008 Zbl 0548.53006

[López and Martín 2001] F. J. López and F. Martín, "Minimal surfaces in a wedge of a slab", Comm. Anal. Geom. 9:4 (2001), 683-723. MR 2002k:53013 Zbl 1026.53002

[López et al. 2000] F. J. López, R. López, and R. Souam, "Maximal surfaces of Riemann type in Lorentz-Minkowski space [3", Michigan Math. J. 47:3 (2000), 469-497. MR 2002c:53009 Zbl 1029.53014

[Marsden and Tipler 1980] J. E. Marsden and F. J. Tipler, "Maximal hypersurfaces and foliations of constant mean curvature in general relativity”, Phys. Rep. 66:3 (1980), 109-139. MR 82d:83048

[Meeks and Rosenberg 1993] W. H. Meeks, III and H. Rosenberg, "The geometry and conformal structure of properly embedded minimal surfaces of finite topology in $\mathbf{R}^{3}$ ", Invent. Math. 114:3 (1993), 625-639. MR 94i:53003 Zbl 0803.53007

[Miklyukov 1992] V. M. Miklyukov, "Maximal tubes and bands in Minkowski space", Mat. Sb. 183:12 (1992), 45-76. In Russian; translated in Russian Acad. Sci. Sb. Math. 77:2 (1994), 427-453. MR 94a:53019 Zbl 0801.53042

[Osserman 1971/72] R. Osserman, "The convex hull property of immersed manifolds", J. Differential Geometry 6 (1971/72), 267-270. MR 45 \#7647 Zbl 0226.53009

[Schoen 1983] R. M. Schoen, "Uniqueness, symmetry, and embeddedness of minimal surfaces", $J$. Differential Geom. 18:4 (1983), 791-809. MR 85f:53011 Zbl 0575.53037

[Umehara and Yamada 2006] M. Umehara and K. Yamada, "Maximal surfaces with singularities in Minkowski space”, Hokkaido Math. J. 35:1 (2006), 13-40. MR 2007a:53018 Zbl 05059482

Received December 15, 2005. 


\section{Rosa MARIA BARREIRO CHAVES}

DEPARTAMENTO DE MATEMÁtICA

INSTITUTO DE MATEMÁtiCA E ESTATÍSTICA

UNIVERSIDAdE DE SÃo PAULO

RUA DO MATÃo, 1010

05508-090 SÃo PAUlo, SP

BRAZIL

rosab@ime.usp.br

http://www.ime.usp.br

\section{LEONOR FERRER}

DEPARTAMENTO DE GEOMETRÍA Y TOPOLOGÍA

UNIVERSIDAD DE GRANADA

18071, GRANADA

SPAIN

lferrer@ugr.es

http://www.ugr.es/ lferrer 